\title{
Reflection of Space-Time in Ancient Cultural Heritage of Urals*
}

\author{
Alina Paranina \\ Herzen State Pedagogical University, St. Petersburg, Russia \\ Email: galina_paranina@mail.ru \\ Received 9 July 2016; accepted 1 August 2016; published 4 August 2016 \\ Copyright (C) 2016 by author and OALib. \\ This work is licensed under the Creative Commons Attribution International License (CC BY). \\ http://creativecommons.org/licenses/by/4.0/

(c) (i) Open Access

\begin{abstract}
Based on the example of Shigir idols, the article reviews the possibility of using the ancient sacred objects as tools for orientation in space-time (according to dating of C-14, the age of the Grand Shigir Idol is 11,000 years). Author's reconstructions of solar navigation technology are justified by: paleo-astronomic and metrological calculations; reflection of curve of shadow of the gnomon during the year in the proportions of the body and face of anthropomorphic figures; comparison of geometric basics of "faces" of Shigir idols, masks of Okunev stelae in Khakassia and vertical sundial on the milestones of the eighteenth century in St. Petersburg. The results show a rational assignment of objects of ancient culture.
\end{abstract}

\section{Keywords}

Ancient Cultural Heritage, Shigir Idols, Okunev Stelae, Sundial and Calendars, Orientation in Space-Time

Subject Areas: Archaeology

\section{Introduction}

Big Shigir Idol is the most ancient wooden sculpture in the world, made of larch in the Mesolithic (radiocarbon age of 11,000 years). It is stored in the Sverdlovsk regional museum in Yekaterinburg (Russia). Unique archaeological object was discovered in 1890 by workmen of Kurinskiy gold mine, at depth of 4 meters of peat deposits of drained part of the lake, on the eastern slope of the Middle Urals. Along with the Big Shigir idol various objects made of bones and wood were found, including Small Shigir Idol (Figure 1). High craftsmanship of the found artifacts is identified by archaeologists as a surge of historical and cultural development. However, the astronomical designation of objects has not yet been considered.

\footnotetext{
*Article was originally published in the edition: Collection of articles of Russian scientific-practical conference. Ministry of Culture and Tourism of the Sverdlovsk region, Sverdlovsk Regional Museum. Institute of History and Archaeology, Ural Branch of RAS. Yekaterinburg SQUARE, 2011-264 p-ISBN 978-5-91357-023-9.
} 


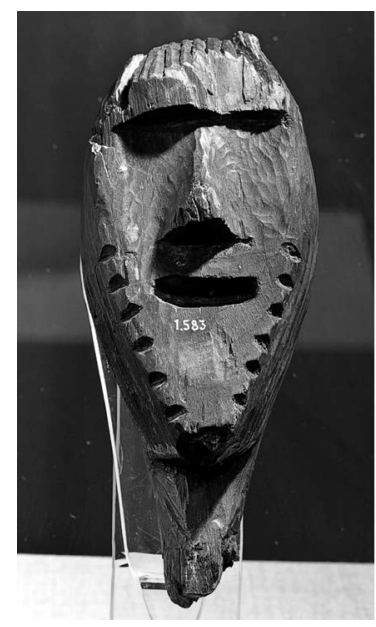

(a)

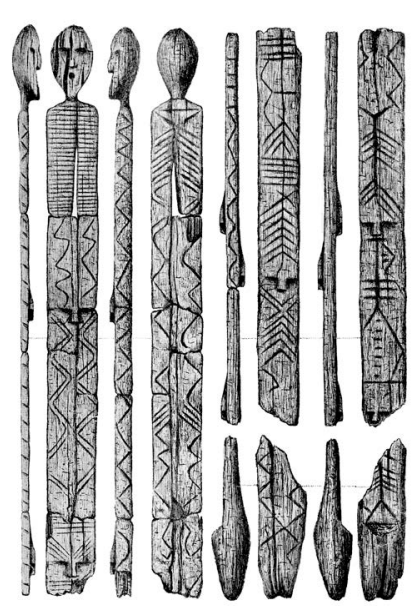

(b)

Figure 1. (a) Small Shigir Idol [1]; (b) Big Shigir Idol [2].

\section{Reflection of Space-Time as a Basis for the Veneration}

Previous research on the semiotics of geographical space dedicated to cyclic dynamics geometry of shadow of the gnomon in the northern labyrinth and other astronomic solar navigation tools of different latitudes of the Earth allows the author to review the issue from the natural-scientific point of view-to what extent do the objects of the ancient cultural heritage of the Urals and other regions of Eurasia (idols, stelae, statues, menhirs and others) reflect the practically important information for humans?

As a methodological basis of functional and semantic analysis of ancient cultural heritage of the Urals the provisions set at different stages of research are considered: 1) about key informational role of sacred natural, natural and artificial and artificial objects in the prehistoric life-support system; 2) about implementation of long-term storage function of navigational information; 3) about information on the possible parameters of the universal position in space-time to determine any object [3].

The instruments for theoretical development of the issue are the main stages of encoding information about cosmo-planetary processes, important for the navigation in space-time. Comparison of annual curve of shadow of the gnomon and known solar signs, mythological images of the sun and the time (Figure 2) allows one to imagine the process of encoding information in the sequence of the "process-sign-image", in other words: 1) geometric symbol is a model of a natural process; 2) the mythological character is a subject-shaped model based on geometrical signs, which directly reflects this process.

In contrast to the geometrical sign, the image is more generalized and abstract with respect to the sourcenatural process, but it is more concrete and understandable for ordinary perception. Dialectical contradiction of this demonstration of general relativity clarifies the objective basis of the problem of interpretation of the primitive graphics and ancient art.

Scheme of annual dynamics of shadows (Figure 2(a)) explains the origins of the different, at first glance, images as six- or many-armed Shiva, sunny cancer, lotus - the symbol of the year, "the day of the gods." A common feature of these images is the conceptual area of their formation associated with common scientific and religious beliefs about the events of time and life. Mystical features of mythological characters can be understood as an attempt to express the breadth and diversity of a not completely investigated, and hence mysterious, natural process.

\section{Evolution of Astronomical Tools and Universal Standards of Space-Time}

We can assume that the main stages of human life became the means of the mythological description of natural processes, just like metrological characteristics of the objects of the world were built on the system of anthropological equivalents.

One of the most well-known ways of using resources of creative thinking, backed by practical convenience and stored in the saying "All that is mine is with me all the time" is the application of space and time of dimen- 


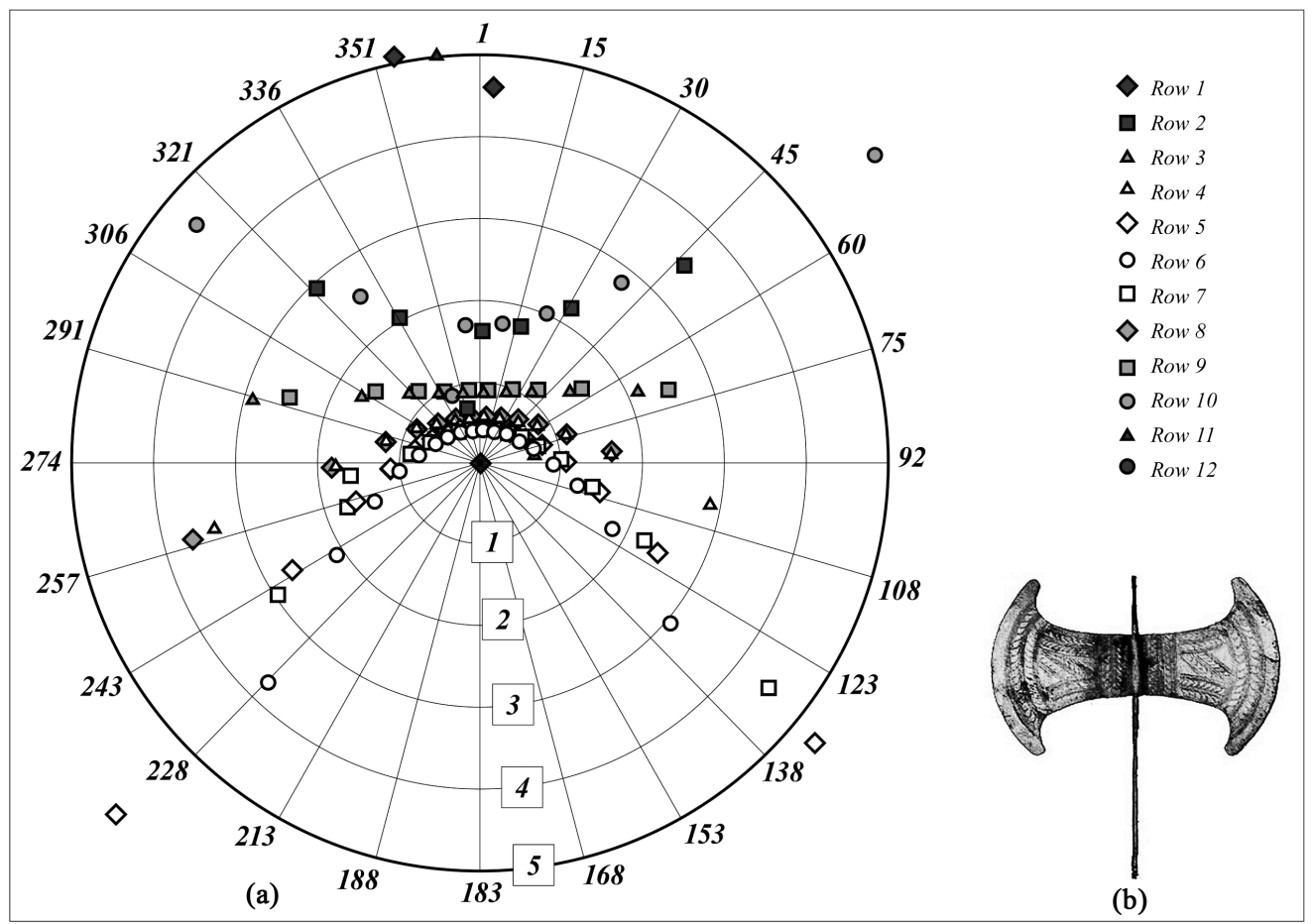

Figure 2. (a) The sum of shadows of the gnomon during the year is in the form of Labrys [3]; (b) Labrys, two-way, two-horned axe of Zeus [4].

sions of the elements of the human figure as metrological equivalents. The best known linear measures are fathom, arshine and their parts (elbow, hand, finger), for the size of the foot (feet) is also known to use in determining the time. According to historical records in Babylon, and ethnographic evidence of the late twentieth century - in Central Russia, the equivalent of hours of the day was the number of feet in length shadows of a standing person. The ancient legend of the labyrinth argues that in the center of the labyrinth God-the creator of the world cut through darkness by one side of the Labrys, and cut himself by the other side and found in his hand is not Labrys, but a torch of fire that actually draws a picture of the indissoluble relationship of light and shadow, with precise localization in the center of the labyrinth. Moreover, the figure is the source of the shadow, as stated in the extant tradition.

Navigation traditions have an affinity not only when regions of Eurasia are compared. Navigation by the Sun has been used by all peoples of the world. In ancient Egypt, the number 64 was used for the account, which is equal to the local value of the azimuth of sunrise at the summer solstice and called "Eye of Horus". Semantic analysis of the myths that reflect the basic ideas of the famous solar cults shows that eyes allegorically reflect visioning - observation of astronomical objects in the sky.

Using the palm as a tool for navigation in space-time was noticed by us in communication with indigenous peoples of village Kolatselga in Karelia, and the descriptions known for Armenia. Subject-gnomon equal in length to one of the fingers is secured perpendicularly to the plane of the palm by pinched fingers and according to position of its shadow the time of the day is determined. We can assume that a well-known mark of the Phoenicians - outline of palm is often found on the cliffs along the ancient sea routes, symbolizing mastery of navigation technology of this type. The version of calendar calculations on the elements of the palms survived nowadays, which became known as the "hand of Damaskin", named after the author, who recorded it first. Among the features of this system-Calculation of the Christian Easter, which takes into account cycles of the sun and the moon. As we see, oral tradition kept a complex knowledge for a long time, the continuity of which probably relied on the link between generations and continuity of cultures.

The description of the Isis in Ancient Egypt used the feature of "knowledge are on her head". At the same time the throne was meant, depicted alone or between the horns. Analysis of the hats and the women's hairstyles in Paleolithic period shows the stability of this tradition: the symbolic role is connected with ringlets, braids, as 
signs of age, and elements of rites of passage (the number of braids, their braiding and unwinding). The custom in Delphi is deeply symbolic according to the description of ancient authors. This custom includes laying a curl on the spindle on the graves of the girls who came from Hyperborea

It is interesting to imagine the evolution of the transfer of human qualities of the surrounding nature and back. Probably, the first step in this direction was the application of size and strength of arm, which own the first role in the tactile exploration of environment. The definition of the height using parts of the body is old as well ("grass to the waist," "sea to a knee”...). The next step would be giving the surrounding objects of sacred standard size and at the same time the artificial objects obtain property of its creator, who created them by his own similarity, and later-by the image. Developing this direction technically, a human came to the ancient classical sculpture, from one side, and to engineering inventions - from the other side, adapting to the role of the creator psychologically — to a level of ambition that allowed to assign to human image the highest divine status.

The simplest astronomical tools-including sundial and calendar, are the product of generalization of properties of the surrounding material. To some extent, it can be seen as an abstraction-a model of the world. But the purpose of the tool is quite definite- to broadcast vitally important navigational information of specific locations in real time.

It is impossible to separate information on space and time, in the modern scientific paradigm this conclusion is reflected in the form of provisions on the time-space continuum, which seems obscure to many, but in ancient times this feature of nature was obvious for everyone in the everyday experience: the noon was determined by the shortest shadow of the gnomon, and at the same time the direction of the geographical north, the names of landmarks of space and time coincide not accidently: the east— the sunrise, west—sunset, "midday and midnight countries"-north and south.

The development and improvement of navigation technology has led to the separation of the functions of navigation tool-magnetic compasses, chronometers were invented. But a tradition of combination of space-time benchmarks reached almost to the present day. Just 200 years ago, when light portable mechanical watches were not used widely, the sundials were installed on milestones, and milestone itself kept a pillar shape of stelesgnomons of all times (Figure 3).

If a rational scheme of a tool gets features of real object according to the basis of geometric similarity or express the similarities and vague associations by means of art, connected with general idea of integral process, then a mixture of the real and the ideal will appear, which carries the notion of "mythological image". Samples of such fantastic anthropo-zoo-morphic images are often found in the ancient heritage of Southern Siberia (Figure 4(a) and Figure 4(b)).

In Khakassia there are many stone statues and steles of Okunevskaya archaeological culture (III-II millennium BC), with carved faces, solar symbols and other images. According to current interpretations in archeology, sculpture (Figure 4(a)) is associated with the central axis of the universe-the epitome of world mountain or sacrificial post-ritual equivalent to the World pillars. Face is considered as an image of primordial essence and spatial system: the eyes, nose and mouth are regarded as its three tiers, and the ears - the parts of the world [5].

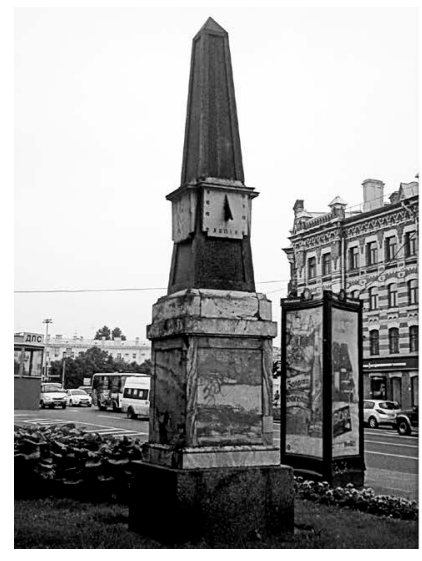

(a)

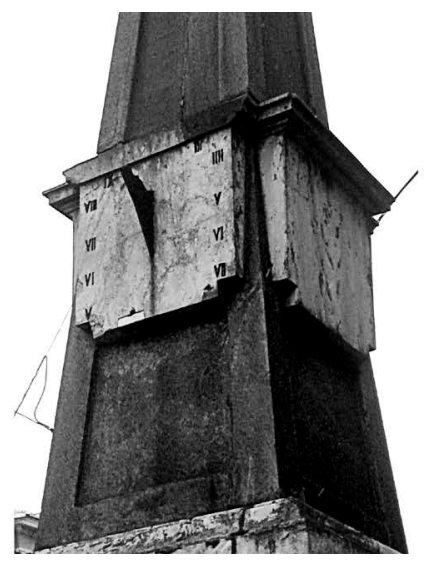

(b)

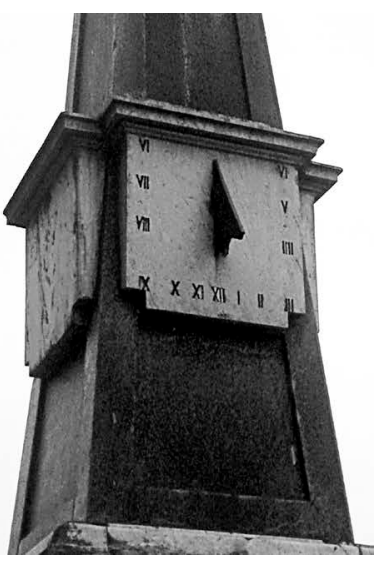

(c)

Figure 3. Vertical gnomons—sundial on the milestones, St. Petersburg Photos of A.N. Paranina. 


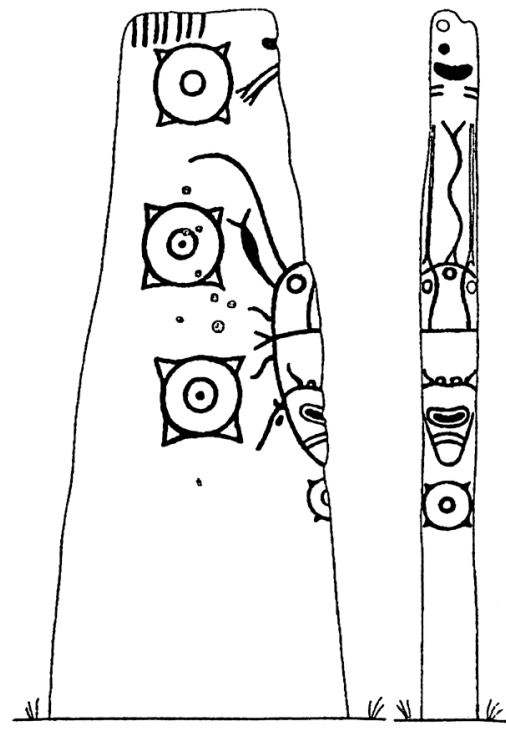

(a)

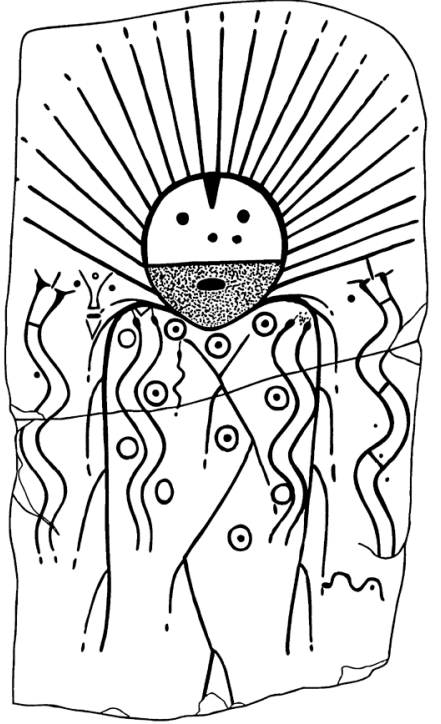

(b)

Figure 4. Stele of Okunevskaya culture in Khakassia: (a) Sculpture with solar signs and "sun horns," (b) Sun-faced stele, drawings by Y. Esin (Y. Esin, 2010).

The use of solar navigation (in the shadow of the gnomon) extends the possibilities for understanding the faces: the lateral horns—symbols of sunrise/sunset, middle horn and the third eye - the image of the gnomon, which always occupies a central position on the observation platform and materializes information link between sky and earth, because the angle of the light beam is encoded by form of shade on the ground [3].

Stele with rays around the "face" are called sun-faced. Comparison of drawing of such stele with the sum shadows of the gnomon (Figure 4(a) and Figure 1(a), where rows are numbers of months of the year) allows to notice similarities: if we consider the horizontal strip on the "face" as a significant equivalent of line of shadows in the equinox, then the mouth will correspond to the position of gnomon, the points of nose and eyes are arranged in an arc-shaped horns facing up, the vectors of which correspond to the shadows at the sighting of sunrise-sunset in winter (the angular distance suggests that the image depicts a solstice). A graphical drawing of vertical sundial can be seen in the divergent rays, where the tooth is a benchmark for accurate determination of the noon.

Snakes are an important element of the considered image, their images are associated with the annual cycle, the knowledge and the universal order. Four snakes can be associated with a number of astronomical seasons (4), two of them are located in the area of drawing, concluded between the descending lines and are connected, in our opinion, with the benchmarks of night sky. It is noteworthy that the snakes, located in the light, have three "horns" on their heads. Association of snake's sting (in other cases-the tongue) with a gnomon is supported by a comparative linguistic analysis—-similar structure "vowel sound- $[\mathrm{s}]-[\mathrm{t}]$ " unites the word mouth (in Russian "usta"), truth (in Russian "istina") and to be-is (ist). The meaning of these notions is the source, the foundation, the time being.

The diversity of the image includes the phenological aspects of the ancient calendar: the snakes in the central (dark) part are deprived not only of a sting but also bands that generally characterize their status as passive, which corresponds to the phase of sleep or anabiosis. Zigzag in this part of the image may indicate the median border of night time and winter season.

In general, our interpretation of solar masks allows to allocate semantic parts associated with annual and diurnal cycle of the sun, light and dark areas in their allegorical parallels with the seasons of summer and winter, and highlight their climax due to phenological calendar.

Apparently, adjusting to the ratio of "physicists" and "poets" in the human population, the coding of navigational information is displayed on the level of object forms, artistic stories, making it publicly available, easy to transfer to younger generations, recognizable in a new geographical environment. Simplicity in memorizing and transferring of knowledge has served as a guarantee of long-term preservation. At the same time, the amount of 
knowledge has been accumulating, which ensured the creation of original designs by the same rules, in conformity with the laws of nature.

\section{Shigir Idols as a Figurative Embodiment of Astronomical Tools}

If you compare the face of the Small Shigir Idol and a sundial (Figure 1 and Figure 2(c)), it is clear that the nose may serve as a gnomon, and the notches on the chin are sectoring for parts of the day. There are only 12, so they can be elements for counting months of the year as well. Similarly, we consider multifunctionality of arcs of labyrinth, which reached brevity of expression and ease of understanding in the 7-arc bi-spiral forms, they include two "snakes"-spirals at the same time: they symbolize halves of the year with the ascending and descending movement of the midday sun and they form 7 arcs—counting elements suitable for observation of 12 months (using 5 medium twice a year) and the seven-day week.

Mouth of Small Shigir Idol is the middle of the day when the shadow passes this part, you can create the illusion of movement of tongue, speech and communication when you are moving. Not coincidentally, the gnomon in ancient Greece and Egypt was called "one who knows", "pointer", which, together with its functions fills the original "nomon", which was inherited by words "number", "nominal."

Big Shigir Idol (Figure 1, on the right) can be considered from the perspective of a combination of broadcast of a real navigational information and reflection of general knowledge about the complete natural process. First of all, when analyzing the image, the statement proved by B.A. Frolov can be used: simple graphical, rhythmically repetitive signs reflect the number (quantitative features of nature) [6]. Indeed, the number of horizontal notches is 29 and those disposed on the neck-31, which is close to the number of days in the lunar or solar month.

Further we will use our conclusion that signs, symbolizing, first of all, time and signs of space, which are inseparable from it, are shaped by the schematic reflection of cyclical processes.

The role of space in ancient times has not been overestimated, which is proved by the formula "everything flows, everything changes" and cosmogonical idea that only Time is eternal, the mastery of which means absolute power. With such a prioritization, meanders resembling river in ancient specimens of art are semantically closer to the snake - the most enduring symbols of time and the calendar (in the labyrinth, in the hands of the Cretan mother goddess, on the head of Egyptian gods and pharaohs, in the garden of Eden and under a hoof of horse of famous saint...). In the ornament of Big Shigir Idol the mating zigzags, resembling river meanders or bends of snakes, form a multiple repetition of the cross-shaped pattern.

A saltire encoding azimuths of solstices, has many examples of the use of the calendar in South Siberia (in the image of hip and shoulder joints of solar bulls on petroglyphs). Azimuth of sunrise at the summer solstice at latitude of Shigir lake 7 - 9 thousand years ago changed within the range of deciles of degree and corresponded to $40^{\circ}$. The drawing shows that such vectors of sunrise/sunset at solstices are used for imaging the front of the head, masks on the torso and their linear frame. Other angles in "chevron" ornaments vary within the calendar year range, except for the corners of the rhombus, which includes a column of seven dashes, which may reflect the day of the week and azimuth of the moon.

Fragments of shape can serve as metrological equivalents of length midday shadow for seasons. According to paleo-astronomical calculations, 9 - 6.5 thousand years ago $57 \mathrm{~N}$ the height of the sun throughout the year ranged from $57^{\circ}$ to $8^{\circ}$, at the equinox it was a little more than $32^{\circ}$. According to the formula $h / \operatorname{tg} a$, where $h$ is the height of the gnomon is equal to $1 \mathrm{~m}$, we get the lengths of the shadow of the gnomon which are $0.65 ; 1.59$ and $7.17 \mathrm{~m}$ (the obtained dimensions are convenient to use for estimating the ratio of height and shadow of the object). At a height of idol of $5.3 \mathrm{~m}$ noon shadows throughout the year will range from 38 to 8 and $3.5 \mathrm{~m}$, and that may be reflected on the surface at the point of its installation. We can assume that the convenient location, well-lighted, noticeable at a big distance and providing a panoramic view of the horizon was chosen on the high bank of the river. In this case, the rapid flood could cause undermining and collapse of the bank, and thus transfer of wooden construction with water away from the site of the original installation.

If the head of the idol is not included in the calendar of lengths of shadow, then on a $5 \mathrm{~m}$ long board all the shadows from the gnomon $\mathrm{H}=0.7 \mathrm{~m}$ can be located, and this size in the old system of measures corresponds to arshine. The shortest shadow of the year is equal to $0.45 \mathrm{~m}$ (elbow), and at equinox it is $1113 \mathrm{~m}$, i.e. 2.5 elbow. All flat part of the figure includes 11.1 of elbow, and its full-height of $5.3 \mathrm{~m}(1.77 \times 3)$ is equal to three national or swinging fathoms (size between the ends of the fingers extended horizontally in opposite sides of the hands) 
—one of the oldest from the survived in ethnographical time.

We can compare the obtained dimensions with a drawing in order to highlight the markers on the surface of idol. Equivalents of summer solstice can be the first and second pieces of the picture in the upper part of the "body", next segment board with double zigzag corresponds to the length of the shadows in the equinox. The boundary of this part of the figure is the "mask" second from the top with nose and eyebrows, above which the lines of drawing reflect direct sighting of azimuths of sunrise/sunset in summer. Below this level, "chevron" drawing is turned up with corners, which reflects the direction of direct sighting in winter. Below is the third "mask" there is a saltire-a symbol of culminations-solstices. On the reverse side of the board a saltire is marked at the top of the figure. In this way, cyclical process and its main internal boundaries, separating ascending and descending movement of the sun in six months can be described.

Location of sacred objects is usually connected with linear forms of the landscape, oriented at an azimuth of sunrise/sunset at the solstice or equinox, marking their importance as reference points of time. In this respect, the outskirts of Shigir lake have not yet been researched.

The connection of navigation facilities, which have always played an important role in the life support system, with the cult of ancestors is obvious-it is evidence of their wisdom and care for grandchildren and great grandchildren, passing through the centuries. The rise of interest in the ancient megalithic heritage, which is noticed throughout nowadays, shows that humanity stands on the edge of spiritual and environmental crisis, now it is the time for apprenticeship.

\section{Conclusions}

Layout and ornamentation of Shigir idols reflect the technology of navigation in space-time by the sun.

Comparative analysis of design of Shigir idols, sundial calendars and objects of ancient cultural heritage of other regions show that similarity of geometric basis sets the invariant of natural process, and a variety of decorative design are regional and epochal preferences.

Research of archaeological sites of the Middle Urals opens new depths of navigational traditions in space-time by the Sun (Mesolithic) and confirms the author's concept of the navigational designation of sacred objects.

\section{References}

[1] http://varandej.livejournal.com/273678.html

[2] http://www.ihist.uran.ru/gallery/articles/shigir_idol.jpg

[3] Paranina, G.N. (2010) Light in the Labyrinth: Time, Space and Information.

[4] http://taina-simvola.ru/labris/

[5] Esin, Y. (2010) The Mystery of the Ancient Gods of the Steppe. Abakan.

[6] Frolov, B.A. (1992) Primitive Graphics of Europe.

Submit or recommend next manuscript to OALib Journal and we will provide best service for you:

- Publication frequency: Monthly

- 9 subject areas of science, technology and medicine

- Fair and rigorous peer-review system

- Fast publication process

- Article promotion in various social networking sites (LinkedIn, Facebook, Twitter, etc.)

- Maximum dissemination of your research work

Submit Your Paper Online: Click Here to Submit

Contact Us: service@oalib.com 